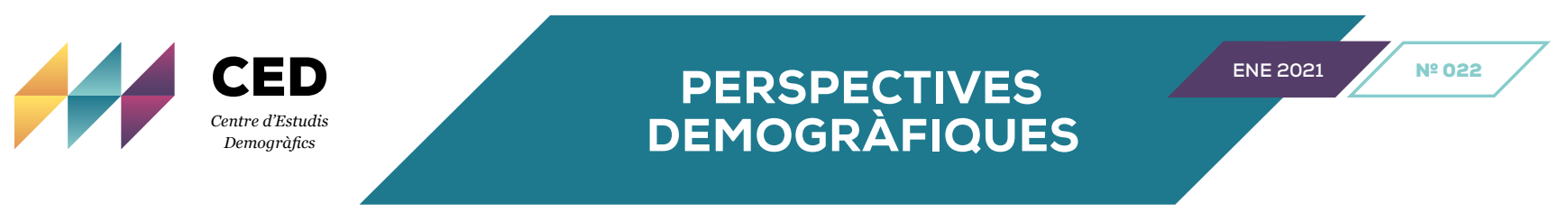

\title{
La esperanza de vida libre de enfermedad no aumenta en España
}

Pilar Zueras, Institute for Social and Economic Research, University of Essex, y Centre d'Estudis Demogràfics y Elisenda Rentería, Centre d'Estudis Demogràfics

La COVID-19 ha supuesto un parón en la continua progresión de la esperanza de vida al nacer, que en España se situaba entre las más altas del mundo. A pesar de que su efecto depresivo sea coyuntural, ya que se espera que mejore de forma rápida en los próximos años, nos ha hecho reflexionar sobre la evolución previa a la pandemia. Es cierto que el aumento de los años de vida ha sido progresivo y continuado, pero en los últimos años estas ganancias no se han traducido en mejoras en la salud entre la población mayor. Aunque la esperanza de vida libre de discapacidad a los 65 años ha sido muy estable en el tiempo, los resultados son menos alentadores cuando se considera la presencia de enfermedades y condiciones crónicas. En este número de Perspectives Demogràfiques investigamos si se han ganado o perdido años de vida libres de enfermedades a la edad de 65 años entre los años 2006, 2012 y 2017 entre los hombres y mujeres en España y por comunidades autónomas (CCAA). Los resultados indican que, a pesar del aumento en años de esperanza de vida, también aumenta el tiempo que las personas viviremos con enfermedades diagnosticadas si no se revierte la tendencia actual. Entre las enfermedades y condiciones más frecuentes están la hipertensión, el dolor de espalda crónico, la diabetes y las enfermedades del corazón. Las diferencias entre hombres y mujeres tienden a converger en años de vida pasados en buena salud como consecuencia, principalmente, del alargamiento de los años de vida vividos en mala salud entre los hombres.

\section{LA ESPERANZA DE VIDA Y EL INCREMENTO DEL TIEMPO VIVIDO CON ENFERMEDADES}

La esperanza de vida en España se encuentra actualmente entre las más altas del mundo con 80,9 años para los hombres y 86,2 años para las mujeres en 2019. Este crecimiento positivo se ha observado sin interrupciones desde la década delos 70 del siglo pasado. Cabe preguntarse, sin embargo, si estas ganancias de vida han sido también en buena salud, especialmente entre las personas mayores. Según los datos del Instituto Nacional de Estadística (INE), la esperanza de vida en buena salud al nacer ha aumentado durante los últimos 15 años. En cambio, los años vividos en buena salud a partir de los 65 años se han mantenido bastante estables a pesar del crecimiento en la esperanza de vida (INE, 2020). La medida más común para definir buena salud se basa en la ausencia de limitaciones

FIGURA 1. Evolución de la esperanza de vida a los 65 años y de los años vividos con y sin enfermedades

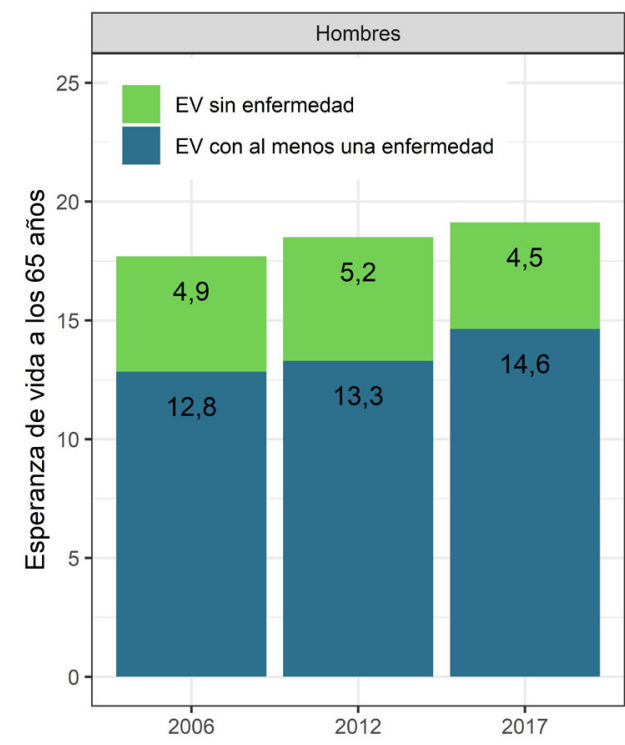

Fuente: INE y ENSE 2006, 2012, 2017

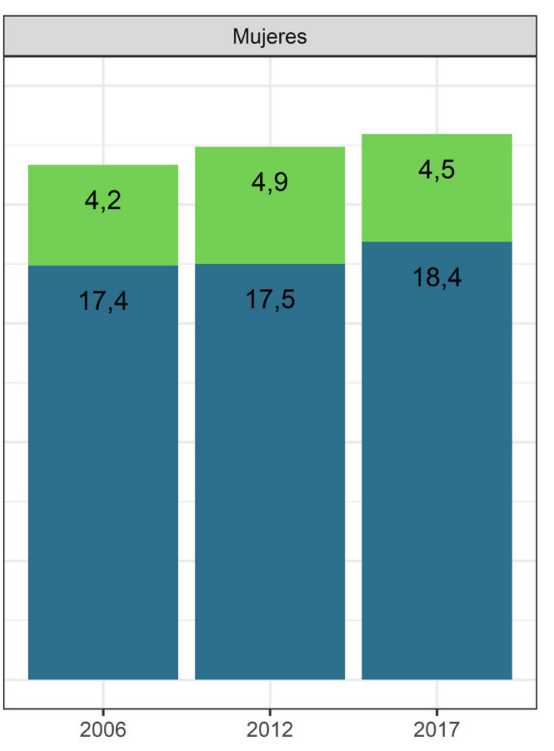

funcionales o discapacidades. Con todo, es importante saber qué pasa con otros indicadores de salud, como, por ejemplo, conocer cuánto tiempo de nuestras vidas pasaremos con enfermedades crónicas o condiciones adversas de salud. Esta perspectiva es relevante porque las enfermedades crónicas son un fuerte determinante de la calidad de vida de las personas y están relacionadas con muchos problemas de salud, aumentan el riesgo de morir más joven y definen las necesidades sanitarias de la población, determinando el nivel de utilización y el tipo de intervenciones que realizan los servicios de salud.

Los aumentos de esperanza de vida 
derivan de mejoras que retrasan la muerte de las personas. $\mathrm{Si}$ al mismo tiempo que se pospone la muerte, la edad en que empiezan las enfermedades o problemas de salud no varía, se produce lo que se llama una extensión de la morbilidad, donde pasamos a vivir más años en mala salud. Pero si la edad en que aparece la enfermedad se retrasa, pueden ocurrir dos cosas. Por una parte, si esta edad aumenta más lentamente que la esperanza de vida resultaría, de nuevo, en la extensión de la morbilidad. En cambio, si la edad de inicio de algún problema de salud se retrasa más rápidamente que el crecimiento de la esperanza de vida provocaría una compresión de la morbilidad, que se concentraría más en los últimos años de vida. Los datos de las encuestas nacionales de salud combinados con los datos de mortalidad por edad nos permiten investigar si ha habido compresión o expansión de la morbilidad en España en los últimos años, es decir, si el tiempo de vida con mala salud ha aumentado o ha disminuido. Así pues, calculamos el tiempo de esperanza de vida a partir de los 65 años que vivimos en España con alguna enfermedad crónica o condición de salud y si se distribuye de manera diferente en las CCAA. Dadas las diferencias en mortalidad y morbilidad entre sexos, analizamos su reciente evolución separadamente para hombres y mujeres. Usamos los datos de las encuestas nacionales de salud de 2006, 2012 y 2017, donde investigamos la presencia de varios problemas de salud como asma, cáncer, diabetes, ictus, hipertensión, infarto de miocardio, enfermedad del corazón, enfermedad obstructiva pulmonar crónica (EPOC) y dolor de espalda crónico - recordando que la mayoría de ellas está relacionada con la mortalidad por COVID-19. Utilizamos el método llamado de Sullivan que se basa en aplicar la proporción de individuos que tienen una de las enfermedades escogidas a una tabla de vida. El cálculo, basado en observaciones específicas por sexo y grupos de edad quinquenal de la presencia de enfermedad y de las defunciones, permite obtener el tiempo de la esperanza de vida (a partir de una determinada edad) vivido con cada una de las condiciones, con al menos una de ellas o sin ninguna de ellas.

La Figura 1 muestra la evolución de la esperanza de vida a los 65 años para hombres y mujeres entre 2006 y 2017 juntamente con la evolución de la esperanza de vida en esta misma edad con al menos alguna de las enfermedades especificadas. La esperanza de vida muestra un crecimiento lineal pasando de 17,7 a 19,1 años para los hombres y de 21,7 a 23,0 años para las mujeres. Al mismo tiempo, el número de años vividos sin enfermedad ha tenido una evolución más estable, donde aumenta ligeramente entre 2006 y 2012 y vuelve a disminuir entre 2012 y 2017, pero manteniéndose siempre en torno a los 5 años para los hombres y ligeramente inferior entre las mujeres. Complementariamente, los años vividos con alguna enfermedad han aumentado a un ritmo más lento entre 2006 y 2012 pero han mostrado un incremento mayor entre 2012 y 2017, especialmente entre los hombres. En cuanto a las diferencias por sexo, las mujeres presentan, de forma consistente, una esperanza de vida superior a los hombres en todos los años. Pero cuando observamos la evolución de los años vividos en buena salud, se constata que ha habido una convergencia entre ambos sexos, donde en 2006 los hombres vivían más años sin ninguna de estas enfermedades, pero en 2017, este tiempo vivido en buena salud se equipara al de las mujeres. De todas formas, como las mujeres tienen una esperanza de vida superior a los hombres, el tiempo vivido con enfermedad es también más largo por ellas durante todo el periodo. A pesar de los años de vida ganados entre 2006 y 2017, 1,3 para los hombres y 1,4 para las mujeres, ellos han perdido 0,4 años libres de enfermedad (-26,7\% del aumento de la esperanza de vida) y ellas han ganado o,3 años (23,9\% de su ganancia). Pero el impacto y la evolución de las enfermedades no ha sido igual para hombres y mujeres.
FIGURA 2. Evolución de la proporción de la esperanza de vida a los 65 años vivida con cada enfermedad por sexo

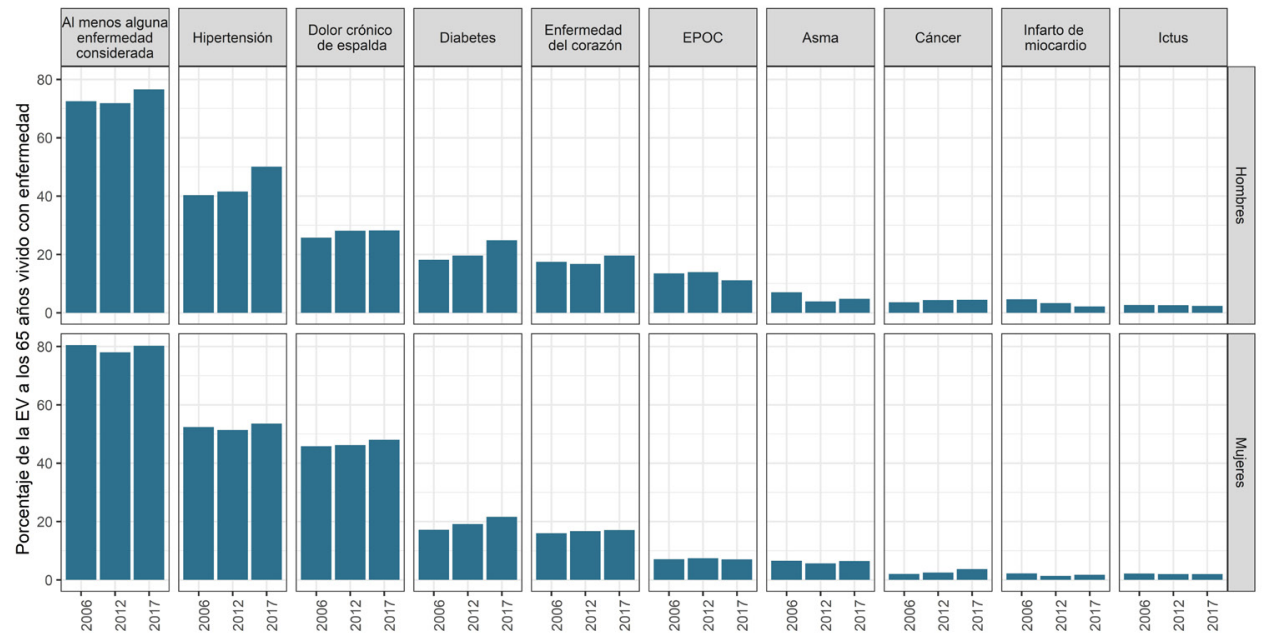

Fuente: INE y ENSE 2006, 2012, 2017 


\section{LAS DIFERENCIAS DE GÉNERO EN EL TIEM- PO VIVIDO CON CADA ENFERMEDAD}

Este estudio incluye nueve condiciones de salud que son especialmente relevantes por diferentes motivos. El cáncer y las enfermedades cardiovasculares y cerebrovasculares (ictus, infarto de miocardio y otras enfermedades del corazón) estaban entre las cinco principales causas de muerte en España durante el período analizado. Además, hemos considerado dos afecciones de salud que son factores de riesgo cardiovascular: hipertensión y diabetes. Hemos incluido el dolor de espalda (dolor lumbar y cervical crónicos), que fue el principal factor asociado a los años de vida vividos con discapacidad en España en 2016. Finalmente, también hemos examinado el asma y la enfermedad pulmonar obstructiva crónica (EPOC), las dos enfermedades respiratorias más frecuentes. Se ha descartado, por el contrario, incluir tener colesterol alto ya que parte del aumento observado es consecuencia del cambio de parámetros de diagnóstico entre las diferentes encuestas. Tampoco se han podido incluir otras enfermedades que causan deterioro funcional (como la artrosis) por falta de comparabilidad entre las encuestas. Algunas de las condiciones de salud consideradas, no sólo son principales causas de mortalidad y carga de enfermedad y discapacidad, sino también factores de riesgo asociados a la gravedad y el ingreso hospitalario en caso de COVID-19. Cuando analizamos cada una de las enfermedades y condiciones de salud por separado, podemos ver en la Figura 2 cuáles son las que más impactan en el tiempo vivido con mala salud y si hay diferencias entre sexos. Tener hipertensión es la condición de salud más común, donde los hombres de más de 65 años vivían 9,6 años con ella en 2017 y 12,3 años las mujeres, lo que representa 53,6\% de y 50,1\% del tiempo que les queda por vivir a los hombres y las mujeres, respectivamente. Tener dolor de espalda crónico es la siguiente condición más prevalente, especialmente para las mujeres, representando $28,2 \%$ de la esperanza de vida a los 65 años de los hombres y 48,0\% de las mujeres en 2017.

En muchas de las enfermedades podemos observar un aumento sostenido del tiempo de esperanza de vida vivido con la enfermedad (hipertensión sólo para los hombres, enfermedad del corazón sólo para las mujeres y dolor crónico de espalda, diabetes y cáncer para los hombres y las mujeres), pero este aumento ha sido especialmente significativo para tener hipertensión y diabetes entre los hombres. La evolución ha sido diferente en otras enfermedades (asma para hombres y mujeres, enfermedad del corazón para hombres e hipertensión e infarto de miocardio para mujeres) donde la proporción de tiempo vivido con enfermedad se ha reducido o mantenido entre 2006 y 2012 pero ha aumentado entre 2012 y 2017. En el caso de la EPOC, ha aumentado en 2012 y ha disminuido en 2017 tanto para hombres como para mujeres. Por último, algunas enfermedades han disminuido su proporción de tiempo de vida vivido con ellas entre 2006 y 2017 (ictus tanto para los hombres como para las mujeres y, para los hombres, también infarto de miocardio). Tanto en el caso de estas últimas condiciones de salud como en la EPOC, se observa una compresión de la morbilidad.

\section{EVOLUCIÓN Y HETEROGENEIDAD ENTRE CCAA: CRUCE DE TRAYECTORIAS ENTRE HOMBRES Y MUJERES}

Tanto la esperanza de vida como los años vividos con buena o mala salud presentan variabilidad a nivel de CCAA. En la Figura 3 se puede ver la distribución de los diferentes indicadores entre las 17 CCAA estudiadas separadas por sexo y año. La evolución de la esperanza de vida es más consistente y es donde se observa menos variación entre regiones, mostrando un aumento progresivo en todas partes. En cuanto a la esperanza de vida con al menos una enfermedad y la esperanza de vida sin enfermedades, la heterogeneidad está presente en todos los años, donde es inferior en 2012 para los hombres y FIGURA 3. Heterogeneidad regional de los cambios en los años de esperanza de vida a los 65 años

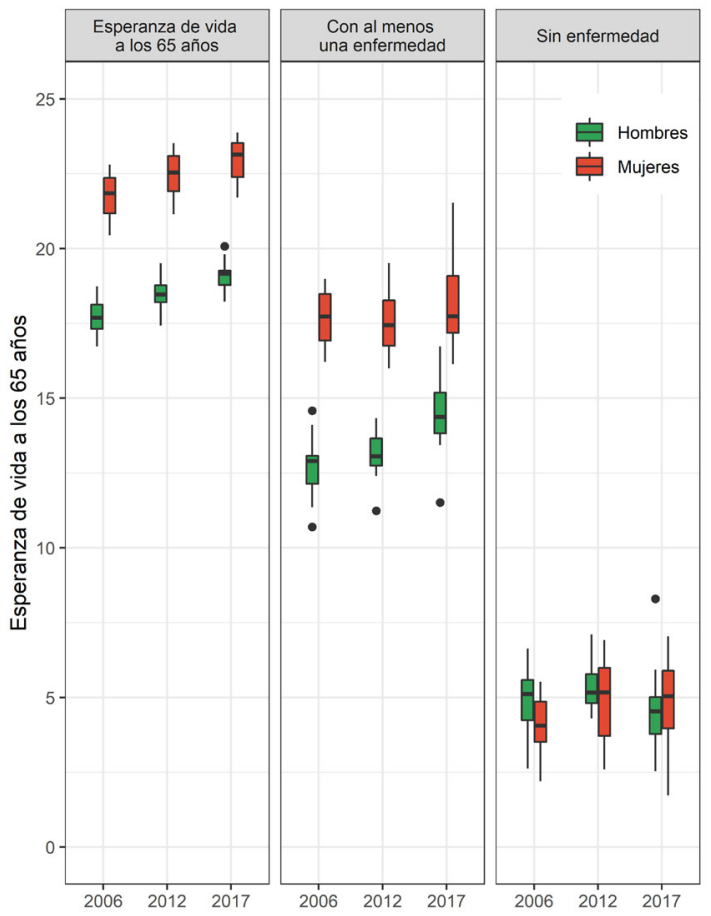

Fuente: INE y ENSE 2006, 2012, 2017

Nota: incluye todas las CCAA excepto las Ciudades Autónomas Ceuta y Melilla. 
en 2006 para las mujeres, pero en los dos casos, 2017 parece el año donde las diferencias entre CCAA han aumentado en mayor medida. Este aumento de la heterogeneidad de la esperanza de vida a los 65 años con alguna enfermedad en 2017 se refleja en una diferencia de valores entre las CCAA de 5,2 años para los hombres y 5,4 años para las mujeres, mientras que en 2006 la diferencia era de sólo 4,0 años para los hombres y 3,3 años entre las mujeres. En muchas CCAA, debido al estancamiento y ligera disminución del tiempo vivido sin enfermedades, especialmente entre los hombres, ha habido un aumento del número de años vividos con alguna enfermedad entre 2006 y 2017. En cuanto a los años vividos en buena salud, la evolución convergente entre sexos lleva a que en 2017 las mujeres equiparen o aventajen los hombres en la mayoría de CCAA, reproduciendo los resultados a nivel nacional.

\section{LA COMPRESIÓN DE LA MORBILIDAD: UN OBJETIVO PRIORITARIO}

Revertir la tendencia a acompañar el alargamiento de la esperanza de vida en las edades más avanzadas con un estancamiento de los años vividos en buena salud debe ser un objetivo prioritario del Sistema de Salud los próximos años. A partir de los 65 años, la presencia de enfermedades y problemas de salud no ha disminuido desde el año 2006, lo que ha provocado un aumento en los años de vida vividos en mala salud, especialmente para los hombres. Tener hipertensión, dolor de espalda crónico, diabetes y enfermedades del corazón son las condiciones más comunes para hombres y mujeres. Y, para la gran mayoría de enfermedades y condiciones consideradas, se ha observado un aumento o estancamiento del tiempo vivido con ellas. Además, este estancamiento en la mejora de los años vividos en buena

\section{Referencias bibliográficas}

Basterra-Gortari, F. J.; Bes-Rastrollo, M.; Ruiz-Canela, M.; Gea, A.; MartinezGonzález, M. Á. (2017) "Prevalence of obesity and diabetes in Spanish adults 1987-2012". Medicina Clínica: 148 (6): 250-256 (DOI: 10.1016/j.medcli.2016.11.022).

INE (2020) Mujeres y Hombres en España, 2020. Salud. 4.2. Esperanza de vida en buena salud [Internet]. 2020 [citado 15 de diciembre de 2020] Enlace

Soriano,, J. B.; Rojas-Rueda, D.; Alonso, J.; Antó, J. M.; Cardona, P.-J.; Fernández, E. et al. (2018) "La carga de enfermedad en España: resultados del Estudio de la Carga Global de las Enfermedades 2016". Medicina Clínica: 151 (5): 171-190 (DOI: 10.1016/j.medcli.2018.05.011).

salud ha sido más pronunciado entre 2012 y 2017, período en el que también se han ampliado las diferencias entre CCAA. Existen factores muy diversos que explican esta tendencia. Por un lado, las mejoras del sistema sanitario se han centrado más en resolver los casos más agudos, más cercanos a la muerte, lo que conlleva una mejora en la supervivencia, pero también un aumento del número de individuos que sufren enfermedades o problemas de salud. Igualmente, durante los últimos años se ha intensificado un empeoramiento de la dieta en toda la población, que, junto con la disminución de la actividad física, provocan un aumento del índice de masa corporal (Basterra-Gortari et al., 2017). Esto tiene, inevitablemente, consecuencias negativas en la prevalencia de enfermedades y condiciones de salud como la hipertensión, la diabetes, enfermedades del corazón, ictus y cáncer. De todas formas, estas tendencias no se han producido de forma similar en el territorio, lo que hace pensar que se pueden implementar políticas que ayuden a mejorar la salud de las personas de edad avanzada. Además, existen importantes diferencias entre hombres y mujeres, destacando que durante los últimos años ellos han sufrido un estancamiento de su salud más pronunciado que ellas. Esta evolución más desfavorable para los hombres que para las mujeres podría estar detrás de la aparente paradoja de la mayor incidencia de severidad y fatalidad por COVID-19 entre la población masculina. Ellas están más expuestas a la infección por su dedicación al trabajo de cuidado, mayor presencia en el sector sanitario y por la propia feminización de la vejez. Sin embargo, el mayor desequilibrio en la cronificación de las enfermedades y la mala salud que acompaña las ganancias de vida en los hombres encontrado en este estudio ayuda a entender, en parte, la mayor mortalidad por COVID-19 entre los hombres que entre las mujeres.

Correspondencia dirigida a

Pilar Zueras

pzueras@ced.uab.es

Elisenda Rentería

erenteria@ced.uab.es

Agradecimientos

Recibimos financiación del Economic and Social Research Council (UK) Research Center on Micro-Social Change de la University of Essex (ES/So12486/1), del Plan Estatal de Investigación Científica y Técnica y de Innovación 20132016. Programa Estatal de Promoción del Talento y su Empleabilida, Subprograma Estatal de Formación y en el Subprograma Estatal de Incorporación: Ayudas para Contratos Ramón y Cajal 2017 (RYC-2017-22586) y del proyecto 'HEALIN' (ERC-2019- CoG-GA No 864616)

\section{Créditos}

Gráficos: Anna Turu

Maquetación: Xavier Ruiz Vilchez
Enlace url

https://ced.uab.cat/es/difusion/butlletiperspectives-demografiques/

Contacto

Centre d'Estudis Demogràfics Calle de Ca n'Altayó, Edificio E2 Universitat Autònoma de Barcelona 08193 Bellaterra / Barcelona España

Teléfono: +34 935813060 E-mail:demog@ced.uab.es Web: https://ced.uab.es/es/

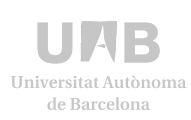

Andreu Domingo y Albert Esteve (Centre d'Estudis Demogràfics) 